\title{
The Impact of COVID-19 on Access to HIV Prevention Services Among Opioid-Dependent Individuals
}

\author{
Colleen B. Mistler ${ }^{1,2}$. Christine M. Curley ${ }^{2,3} \cdot$ Aviana O. Rosen $^{1,2} \cdot$ Renee El-Krab ${ }^{2,3} \cdot$ Jeffrey A. Wickersham ${ }^{2,4}$. \\ Michael M. Copenhaver ${ }^{1,2} \cdot$ Antoine Khati ${ }^{1} \cdot$ Roman Shrestha ${ }^{1,2}$
}

Accepted: 13 March 2021 / Published online: 26 March 2021

(c) The Author(s), under exclusive licence to Springer Science+Business Media, LLC, part of Springer Nature 2021

\begin{abstract}
The COVID-19 pandemic has necessitated restrictive orders and programmatic changes that may be associated with disruptions in services, including those for opioid-dependent people who inject drugs (PWID). This study aims to assess the impact of COVID-19 pandemic on access to and utilization of various HIV prevention services among PWID with opioid use disorder (OUD). We interviewed 110 PWID enrolled in medication for opioid use disorder (MOUD) treatment (e.g., methadone) between May and October, 2020 to identify if this sample experienced changed in access to the following services due to the COVID-19 pandemic: (a) HIV or sexually transmitted infection (STI) testing, (b) pre-exposure prophylaxis (PrEP) services, (c) HIV counselor or doctor appointments, and (d) clean injection equipment. A majority of the sample reported that COVID-19 had not changed their access to HIV testing or access to STI testing. Almost half of the sample reported that getting an appointment with a doctor decreased due to COVID-19. Participants reported that access to a lab or blood testing, access to injection equipment, and sessions with a case manager or counselor decreased. One-fourth of the 32 participants who were taking PrEP before the onset of COVID-19 reported that they had trouble getting their PrEP prescription due to COVID-19, and some reported that they had difficulty getting the PrEP prescription filled at their pharmacy. Our results indicate that PWID did not experience reduced access to HIV or STI testing, but difficulties in obtaining appointments with HIV counselors or doctors and limited access to PrEP were presented. Innovative strategies are needed to reduce the adverse effects of COVID-19 on HIV prevention among PWID.
\end{abstract}

Keywords HIV prevention · Opioid use disorder $\cdot$ Pre-exposure prophylaxis $\cdot$ People who inject drugs $\cdot$ COVID-19

\section{Introduction}

The first case of SARS-CoV-2 (COVID-19) in the United States was identified in January 2020, and as of February 2021, there are over 26 million reported cases and short of

Colleen B. Mistler

colleen.mistler@uconn.edu

1 Department of Allied Health Sciences, University of Connecticut, 358 Mansfield Road, Unit 1101, Storrs, CT 06269-1101, USA

2 Institute for Collaboration on Health, Intervention, and Policy (InCHIP), University of Connecticut, Storrs, CT, USA

3 Department of Psychological Sciences, University of Connecticut, Storrs, CT, USA

4 Department of Internal Medicine, Section of Infectious Diseases, Yale University School of Medicine, New Haven, CT, USA
500,000 COVID-19 related deaths in the United States alone [1]. Most cases have been reported in urban, highly populated areas, though some populations with limited access to testing and treatment infrastructure are at particularly elevated risk, such as opioid-dependent individuals [2-4]. Additionally, individuals with opioid use disorder (OUD) tend to have decreased access to health care, higher prevalence of housing insecurity [5], and an increased likelihood of incarceration [6] compared to those in the general population, and these circumstances pose unique challenges regarding the risks associated with the COVID-19 pandemic [7].

Prior to the spread of COVID-19, the opioid epidemic reignited the prevalence of HIV among people who inject drugs (PWID), as one in ten new HIV diagnoses in the U.S. is among PWID [8]. Unfortunately, limited hours of operation and closures of HIV prevention and drug treatment services (i.e., syringe exchange programs, prescribing medications for HIV prevention) have had deleterious effects 
on accessibility among PWID [7, 9, 10]. Supply chain disruptions, staff absenteeism due to the virus, and fluctuations in facilities' occupancy also limited healthcare access [11]. Pre-exposure prophylaxis (PrEP) is one such HIV prevention strategy offered for PWID in addiction treatment centers that was limited due to the unintended impacts of mitigation strategies to curb COVID-19 cases [12]. Other important medical COVID-19-unrelated services have been largely under-utilized by the patient population as well [13-15].

Moreover, restrictive changes subsequent to the pandemic have exacerbated pre-existing barriers to healthcare (i.e., transportation, stigma and lack of insurance) $[7,16]$. These restrictions were shown to reside primarily at the clinician side as evidenced by the lack of appointments and physician availability, without largely being related to financial reasons such as insurance coverage [17]. This goes hand in hand with previous recommendations at the healthcare level to delay elective care at the expense of urgent visits in order to limit the spread of the virus [18].

Characterizing the impacts of COVID-19 on individuals with OUD is essential to understanding how PWID are uniquely affected by this pandemic and the COVID-19 responses. Furthermore, there is a need to examine whether sub-populations of those with OUD in urban areas are disproportionately impacted by COVID-19, such as racialethnic and socioeconomic minorities [19]. We sought to fill the gaps on the impacts of the COVID-19 pandemic on this vulnerable population by conducting a study among a sample of individuals with OUD enrolled in medication for opioid use disorder (MOUD). This study aims to identify if among a sample of individuals with OUD, access to the following has changed due to the COVID-19 pandemic: (a) HIV or sexually transmitted infection (STI) testing, (b) PrEP prescription refills, (c) HIV counselor or doctor appointments, and (d) clean injection equipment. Based on the evidence of significant disruptions in HIV preventive care and services due to COVID-19-related closures and restrictions, we hypothesized that access to HIV or STI testing, PrEP, and doctor or counselor appointments would decrease. Such findings are necessary to help inform strategies to alleviate these detrimental impacts among this at-risk group.

\section{Methods}

\section{Participants}

The sample was recruited from prior participants of a parent study that included 234 patients from a methadone clinic, assessing their preference for various HIV prevention modalities. Individuals were eligible if they were: (a) 18 years or older; (b) self-reported HIV-uninfected or unknown HIV status; (c) reported drug- (i.e., sharing of injection equipment) or sex-related (i.e., condomless sex) HIV risk in the past 6 months; (d) met DSM-5 criteria for OUD; (e) on MMT; and (f) able to understand, speak, and read English. All patients were treated with methadone for opioid dependence. After the onset of the COVID-19 pandemic (between May and October 2020), we contacted all 234 participants from the parent study to offer participation in a brief survey to understand the impact of COVID-19 on their general wellbeing, sexual health, and access to HIV prevention services.

\section{Study Setting and Procedures}

Participants in the parent study were recruited from an addiction treatment setting in New Haven, Connecticut, via clinic-based advertisements and flyers, word-of-mouth, and direct referral from counselors. For the current study, all participants were contacted by phone and asked to participate in a survey to examine the impact of the COVID-19 pandemic. Flyers were also distributed to subjects whose contact information was out of date. A total of 110 participants completed the 10-min COVID-19-related survey by phone after providing verbal consent. All participants were provided an additional $\$ 10$ for their participation in the telephone survey. The study protocol was approved by the Institutional Review Board at the University of Connecticut and received board approval from the APT Foundation Inc.

\section{Measures}

Demographic characteristics, including age, sex, sexual orientation, ethnicity, marital status, educational status, employment status, annual income, housing status, healthcare engagement, and current methadone dose, were collected. We measured COVID-19 related impacts on healthrelated characteristics, access to HIV services (i.e., HIV and STI testing, PrEP access, HIV counselor or doctor appointments), and sexual health behaviors. Participants were instructed to identify if they had increases, decreases, or no changes in behavior and/or experiences since the onset of COVID-19.

\section{Statistical Analysis}

Statistical analyses were conducted using SPSS v. 25 [20]. We used Chi-squared analysis to research differences in outcomes by demographic variables, including racial-ethnic identity, age, gender, and sexual identity. The significance level was set to $p<0.05$, and we found no statistical differences in outcomes between groups. The presented results in this study are descriptive. 


\section{Results}

One hundred and ten participants completed the study and were, on average, $44.7( \pm 10.6)$ years old. As shown in Table 1 , most of the participants identified as female $(n=62,56.4 \%)$ and White $(n=66,60 \%)$. The remaining participants were African American or Black $(n=26$, $23.6 \%)$, Hispanic or Latino $(n=16,14.5 \%)$, and $2(1.8 \%)$ participants identified as something else. Additionally, most participants identified as Heterosexual $(\mathrm{n}=83,75.5 \%)$, with only 5 (4.5\%) identifying as Gay or Lesbian, $20(18.2 \%)$ identifying as Bisexual, and $2(1.8 \%)$ of participants identified as Other. Less than $10 \%$ were currently employed; 50 participants (45.5\%) were unemployed, and 41 (37.3\%) were disabled or retired. Further, 78 participants $(70.9 \%)$ reported their income was less than $\$ 9999$. Most of the participants $(n=81,73.6 \%)$ were married or living with a partner, whereas $41(37.3 \%)$ were currently homeless. Almost all of the participants had visited a primary care provider in the past 12 months $(n=100,90.9 \%)$, and $73(66.4 \%)$ visited a psychiatrist in the past 12 months. Finally, more than half of the sample reported having Alcohol Use Disorder $(n=64$, $58.2 \%)$ and moderate to severe Depression $(\mathrm{n}=66,60 \%)$.

Overall, the onset of COVID-19 did not have a substantial adverse influence on HIV and STI testing (Table 2). Specifically, 95 of the 110 participants $(86.4 \%)$ reported that the onset of COVID-19 had not changed their access to HIV testing; however, 13 participants (11.8\%) reported that it decreased access to HIV testing. Although 85 participants did not attempt to get an HIV test, 22 of the remaining 25 participants reported that they did not have trouble getting tested for HIV because of COVID-19. Similarly, 90 participants $(81.8 \%)$ reported that the onset of COVID-19 had not changed their access to STI testing; 17 (15.5\%) reported that STI testing decreased. Eighty-nine participants did not attempt to get an STI test, however, 19 of the remaining 21 participants did not have trouble getting STI tested because of COVID-19.

Although the onset of COVID-19 did not ultimately negatively affect HIV and STI testing, it did influence access to care more broadly; 48 (41.8\%) participants reported that getting an appointment with a doctor decreased after the onset of COVID-19. Additionally, participants reported that access to a lab or blood testing (22.7\%), access to injection equipment $(11.8 \%)$, and sessions with a case manager or counselor $(20 \%)$ decreased. One-fourth of the 32 participants who were taking PrEP before the onset of COVID-19 reported that they had trouble getting their PrEP prescription due to COVID-19, and $15.6 \%$ reported that they had difficulty getting the PrEP prescription filled at their pharmacy. Out of the entire sample, $20 \%$ reported that they stopped using PrEP since the COVID-19 pandemic began.

\section{Discussion}

COVID-19 has disproportionately impacted persons in densely populated areas, especially those with chronic conditions, OUD, and racial-ethnic minorities, who have reduced access to health care services $[2-4,19]$. The present study examined the extent to which these sociodemographic characteristics have similarly affected individuals with OUD and PWID regarding HIV/STI testing, availability of PrEP, and ability to secure appointments with health care professionals. Our results indicate that most participants did not report reduced access to blood testing or injection equipment due to COVID-19. Contrarily, almost half of the individuals surveyed expressed difficulties in obtaining appointments with HIV counselors or doctors, which is troubling as these are critical resources for treatment maintenance among PWID. Our hypotheses were partially supported, indicating that structural improvements related to counselor and doctor appointments require drastic improvements but that testing services to this population have not been completely disrupted by the COVID-19 pandemic.

Surprisingly, within this sample of persons enrolled in MOUD, the vast majority reported that for those who desired to test, access to HIV or STI testing had not changed because of COVID-19 restrictions, with less than $16 \%$ reporting decreased access to either testing. Although the need for HVI or STI testing may have been reduced due to social isolation caused by COVID-19 restrictions, the availability of stable testing resources is nevertheless significant $[7,16]$. On the other hand, concomitant antiretroviral therapy- (ART) non-adherence and decreased confidence in attending HIV follow-up visits have also been reported to be growing [21]. Rising evidence is also corroborating the potential surge in opioid overdose among PWID and decrease in bystander rescue during the pandemic [21-23] which can also potentially be due to medication unavailability. This pool of dynamics, coupled with the closure of substance abuse clinics and the reallocation of services to support COVID-19 efforts, could result in deleterious effects on HIV prevention efforts and HIV transmission [21].

The results pertaining to access to PrEP were less optimistic, with one-quarter of individuals on PrEP reporting difficulty getting a prescription from their provider and, to a lesser extent, filling prescriptions at their local pharmacy. These factors may have contributed to individuals deciding to stop taking PrEP. However, it is equally likely that individuals perceived themselves at lower risk during this time frame due to less risky sexual behaviors, less casual sex, fewer sexual partners, and less group sex because of COVID isolation and quarantine efforts [24].

In the context of ongoing challenges in harm reduction programs such as the closure of syringe service programs 
Table 1 Demographics and medical conditions/care

\begin{tabular}{|c|c|c|}
\hline \multirow[t]{2}{*}{ Demographics } & \multicolumn{2}{|c|}{ Total sample $(\mathrm{N}=110)$} \\
\hline & $\mathrm{M}$ & $( \pm \mathrm{SD})$ \\
\hline Age: mean (SD), years & 44.74 & $(0.6)$ \\
\hline \multirow[t]{2}{*}{ Methadone dose: mean $( \pm \mathrm{SD}), \mathrm{mg}$} & 81.36 & $(28.1)$ \\
\hline & $\mathrm{n}$ & $(\%)$ \\
\hline \multicolumn{3}{|l|}{ Gender } \\
\hline Male & 48 & $(43.6)$ \\
\hline Female & 62 & $(56.4)$ \\
\hline \multicolumn{3}{|l|}{ Ethnicity } \\
\hline White & 66 & $(60.0)$ \\
\hline African American or Black & 26 & $(23.6)$ \\
\hline Hispanic or Latino & 16 & $(14.5)$ \\
\hline Other & 2 & $(1.8)$ \\
\hline \multicolumn{3}{|l|}{ Sexual identity } \\
\hline Heterosexual & 83 & $(75.5)$ \\
\hline Gay or Lesbian & 5 & $(4.5)$ \\
\hline Bisexual & 20 & $(18.2)$ \\
\hline Other & 2 & $(1.8)$ \\
\hline \multicolumn{3}{|l|}{ High school graduate } \\
\hline No & 27 & $(24.5)$ \\
\hline Yes & 83 & $(75.5)$ \\
\hline \multicolumn{3}{|l|}{ Current employment status } \\
\hline Employed & 9 & $(8.2)$ \\
\hline Unemployed & 50 & $(45.5)$ \\
\hline Disabled/retired & 41 & $(37.3)$ \\
\hline Other & 10 & $(9.1)$ \\
\hline \multicolumn{3}{|l|}{ English language speaking } \\
\hline No & 5 & $(4.5)$ \\
\hline Yes & 105 & $(95.5)$ \\
\hline \multicolumn{3}{|l|}{ Income level } \\
\hline$<\$ 9,999$ & 78 & $(70.9)$ \\
\hline$\geq \$ 10,000$ & 32 & $(29.1)$ \\
\hline \multicolumn{3}{|l|}{ Living arrangements } \\
\hline \multicolumn{3}{|l|}{ Currently married/living with partner } \\
\hline No & 29 & $(26.4)$ \\
\hline Yes & 81 & $(73.6)$ \\
\hline \multicolumn{3}{|l|}{ Currently homeless } \\
\hline No & 69 & $(62.7)$ \\
\hline Yes & 41 & $(37.3)$ \\
\hline \multicolumn{3}{|l|}{ Clinical outcomes } \\
\hline \multicolumn{3}{|c|}{ Visited primary care provider (past 12 months) } \\
\hline No & 10 & $(9.1)$ \\
\hline Yes & 100 & $(90.9)$ \\
\hline \multicolumn{3}{|l|}{ Visited psychiatrist (past 12 months) } \\
\hline No & 37 & $(33.6)$ \\
\hline Yes & 73 & $(66.4)$ \\
\hline \multicolumn{3}{|l|}{ Alcohol use disorder } \\
\hline No & 46 & $(41.8)$ \\
\hline Yes & 64 & $(58.2)$ \\
\hline
\end{tabular}


Table 1 (continued)

Moderate/severe depression

No

(SSPs) or significant reductions in SSP working hours [25], PWID are at increased risk for HIV transmission and opioid overdoses that could be lethal, as discussed earlier. As in-person meetings with medical providers are limited during the COVID-19 pandemic, mHealth interventions could constitute a suitable alternative to deliver care to PLWH and patients with OUD as they have a high level of satisfaction from both patient and provider perspectives $[21,26]$. Telehealth services should therefore be encouraged when available and amenable to be delivered in an appropriate manner [27]. Although more efforts are still needed in order to develop strategies that would maintain access to care to this population during this pandemic [21], one particular area in which telemedicine could be especially useful is in the partnering with non-clinical community-based service providers which can in turn improve access to care for those patients who do not seek care at the clinic as often as others.

Potential improvement in HIV and OUD services can also be implemented at the MOUD clinic level. Counselors and providers can be educated about the arising HIV transmission and opioid overdose risks, and about the importance of testing and referral to harm reduction services. Providers can also help patients plan for HIV testing by suggesting mobile clinic locations for example or rapid testing.

Finally, although we analyzed the data for differences between gender, age, sexual identity, and racial-ethnic identity, we found none, which is counter to recent studies demonstrating significant disparities along racial/ethnic lines, at least concerning COVID 19 related services and outcomes [19]. It is plausible our contrasting findings may be a result of the fact that our participants are in MOUD, and accordingly, avenues to treatment were established prior to the COVID-19 pandemic, minimizing differences observed in other studies of sociodemographic risk factors and COVID-19 [13].

While this study provides valuable and informative data from a vulnerable and hard-to-reach population, it does have limitations. This study is limited by its sample size and the targeting of PWID who are undergoing treatment. Consequently, access to services for persons not already in treatment cannot be ascertained. In addition, although participants were surveyed by phone, no open-ended responses regarding access or lack thereof were qualitatively analyzed. Lastly, sampling bias may be present due to the time frame the surveys were completed (May-October 2020), in which COVID-19 restrictions may have varied.

The present study adds to the knowledge base for ensuring against gaps in health services for PWID and persons with OUD by identifying the areas with more dire need. While HIV/STI testing and access to PrEP remain important, increasing telehealth appointments for both HIV counselors, case managers, and doctors to help these individuals remain in MMT is paramount. 
Table 2 Access to HIV/STI testing and PrEP

\begin{tabular}{|c|c|c|}
\hline & \multicolumn{2}{|c|}{$(\mathrm{N}=110)$} \\
\hline & $\mathrm{n}$ & $(\%)$ \\
\hline \multicolumn{3}{|l|}{ Access to HIV testing } \\
\hline Decreased & 13 & $(11.8)$ \\
\hline Not changed & 95 & $(86.4)$ \\
\hline Increased & 2 & $(1.8)$ \\
\hline \multicolumn{3}{|c|}{ Had trouble getting an HIV test because of COVID-19 } \\
\hline No & 22 & $(20.0)$ \\
\hline Yes & 3 & $(2.7)$ \\
\hline I haven't tried to get an HIV test & 85 & $(77.3)$ \\
\hline \multicolumn{3}{|c|}{ Had trouble getting an STI test because of COVID-19 } \\
\hline No & 19 & $(17.3)$ \\
\hline Yes & 2 & $(1.8)$ \\
\hline I haven't tried to get an STI test & 89 & $(80.9)$ \\
\hline \multicolumn{3}{|l|}{ Getting tested for STI } \\
\hline Decreased & 17 & $(15.5)$ \\
\hline Not changed & 90 & $(81.8)$ \\
\hline Increased & 3 & $(2.7)$ \\
\hline \multicolumn{3}{|l|}{ Getting an appointment with a doctor } \\
\hline Decreased & 48 & $(41.8)$ \\
\hline Not changed & 59 & $(53.6)$ \\
\hline Increased & 5 & $(4.5)$ \\
\hline \multicolumn{3}{|l|}{ Access to lab/blood test } \\
\hline Decreased & 25 & $(22.7)$ \\
\hline Not changed & 83 & $(75.5)$ \\
\hline Increased & 2 & $(1.8)$ \\
\hline \multicolumn{3}{|l|}{ Sessions with case manager/counselor } \\
\hline Decreased & 22 & $(20.0)$ \\
\hline Not changed & 83 & $(75.5)$ \\
\hline Increased & 5 & $(4.5)$ \\
\hline \multicolumn{3}{|l|}{ Access to injection equipment } \\
\hline Decreased & 13 & $(11.8)$ \\
\hline Not changed & 96 & $(87.3)$ \\
\hline Increased & 1 & $(0.9)$ \\
\hline \multicolumn{3}{|c|}{ Were you taking PrEP before the COVID-10 pandemic? } \\
\hline No & 78 & $(70.9)$ \\
\hline Yes & 32 & $(29.1)$ \\
\hline \multicolumn{3}{|c|}{ Have you had trouble getting your PrEP prescription because of COVID-19? } \\
\hline No & 14 & $(43.8)$ \\
\hline Yes & 8 & $(25.0)$ \\
\hline I haven't tried to get my PrEP prescription & 10 & $(31.1)$ \\
\hline \multicolumn{3}{|c|}{ Have you had trouble getting your PrEP prescription filled at the pharmacy because of COVID-19? } \\
\hline No & 15 & $(46.9)$ \\
\hline Yes & 5 & $(15.6)$ \\
\hline I haven't tried to get my PrEP prescription & 12 & $(37.5)$ \\
\hline \multicolumn{3}{|c|}{ How has your PrEP use changed during the COVID-19 pandemic? } \\
\hline I have never used PrEP & 58 & $(52.7)$ \\
\hline I used PrEP more & 2 & $(1.8)$ \\
\hline I used PrEP less & 3 & $(2.7)$ \\
\hline I have stopped using PrEP & 22 & $(20.0)$ \\
\hline My PrEP use has not changed & 25 & $(22.7)$ \\
\hline
\end{tabular}


Funding This work was supported by grants from the National Institute on Drug Abuse (K01 DA051346 for RS, R21 DA051934 for RS, K24DA051344 for MMC, and K01 DA038529 for JAW) and Grant from the National Institute of Mental Health (T32MH074387-15 for CBM, AOR, CMC, and RE).

\section{Declarations}

Conflict of interest The authors have no conflict of interest to disclose.

\section{References}

1. Centers for Disease Control and Prevention. (2021). COVID data tracker.

2. Centers for Disease Control and Prevention. (2020). COVID-19 in racial and ethnic minority groups. Retrieved July 6, 2020 from https://www.cdc.gov/coronavirus/2019-ncov/need-extra-preca utions/racial-ethnic-minorities.html

3. Centers for Disease Control and Prevention. (2020). Coronavirus disease 2019 (COVID-19). Retrieved July 6, 2020 from https:// www.cdc.gov/coronavirus/2019-ncov/cases-updates/cases-in-us. html

4. Substance Abuse and Mental Health Services Administration. (2020). COVID-19 and opioid treatment programs. Retrieved August 10, 2020 from https://www.samhsa.gov/sites/default/files/ sample-otp-covid-19-faqs.pdf

5. Chatterjee, A., Yu, E. J., \& Tishberg, L. (2018). Exploring opioid use disorder, its impact, and treatment among individuals experiencing homelessness as part of a family. Drug and Alcohol Dependence, 188, 161-168.

6. U.S. Department of Justice. (2017). Drug use, dependence, and abuse among state prisoners and jail inmates, 2007-2009. Retrieved August 10, 2020 from https://www.bjs.gov/content/pub/ pdf/dudaspji0709.pdf

7. Schimmel, J., \& Manini, A. F. (2020). Opioid use disorder and COVID-19: Biological plausibility for worsened outcomes. Substance Use \& Misuse, 55(11), 1900-1901.

8. Centers for Disease Control and Prevention. (2020). HIV and people who inject drugs. Retrieved September 29, 2020 https://www. cdc.gov/hiv/group/hiv-idu.html

9. Davis, C. S., \& Samuels, E. A. (2020). Opioid policy changes during the COVID-19 pandemic-And beyond. Journal of Addiction Medicine, 14(4), e4-e5.

10. Alexander, G. C., et al. (2020). An epidemic in the midst of a pandemic: Opioid use disorder and COVID-19. Annals of Internal Medicine, 173(1), 57-58.

11. Prevention, C.F.D.C.A. (2020). Healthcare facilities: Managing operations during the COVID-19 pandemic. Retrieved January 19, 2021 from https://www.cdc.gov/coronavirus/2019-ncov/hcp/ guidance-hcf.html\#outpatient-ambulatory

12. Mermin, J., PrEP During COVID-19: 5 Things to Know. 2020: Medscape.
13. De Filippo, O., et al. (2020). Reduced rate of hospital admissions for ACS during Covid-19 outbreak in northern Italy. New England Journal of Medicine, 383(1), 88-89.

14. Guo, H., et al. (2020). The impact of the COVID-19 epidemic on the utilization of emergency dental services. Journal of Dental Sciences, 15(4), 564-567.

15. Metzler, B., et al. (2020). Decline of acute coronary syndrome admissions in Austria since the outbreak of COVID-19: The pandemic response causes cardiac collateral damage. European Heart Journal, 41(19), 1852-1853.

16. Kar, S. K., et al. (2020). COVID-19 pandemic and addiction: Current problems and future concerns. Asian Journal of Psychiatry, 51,102064

17. Findling, M. G., Blendon, R. J., \& Benson, J. M. (2020). Delayed care with harmful health consequences-Reported experiences from national surveys during coronavirus disease 2019. JAMA Health Forum, 1(12), e201463.

18. Prevention, C.F.D.C.A. (2020). Framework for healthcare systems providing non-COVID-19 clinical care during the COVID-19 pandemic. Retrieved July 6, 2020 from https://www.cdc.gov/coron avirus/2019-ncov/hcp/framework-non-COVID-care.html

19. Karmakar, M., Lantz, P. M., \& Tipirneni, R. (2021). Association of social and demographic factors with COVID-19 incidence and death rates in the US. JAMA Network Open, 4(1), e2036462.

20. Corporation, I. B. M. (2017). IBM SPSS statistics for windows. . Armonk, NY: IBM Corp.

21. Hochstatter, K. R., et al. (2021). Potential influences of the COVID-19 pandemic on drug use and HIV care among people living with HIV and substance use disorders: Experience from a pilot mHealth intervention. AIDS and Behavior, 25(2), 354-359.

22. Slavova, S., et al. (2020). Signal of increased opioid overdose during COVID-19 from emergency medical services data. Drug and Alcohol Dependence, 214, 108176.

23. Grebely, J., Cerdá, M., \& Rhodes, T. (2020). COVID-19 and the health of people who use drugs: What is and what could be? The International Journal on Drug Policy, 83, 102958.

24. Chow, E., et al. (2020). Changing the use of HIV pre-exposure prophylaxis among men who have sex with men during the COVID-19 pandemic in Melbourne, Australia. Open Forum Infectious Diseases. https://doi.org/10.1093/ofid/ofaa275.

25. Glick, S. N., et al. (2020). The impact of COVID-19 on syringe services programs in the United States. AIDS and Behavior, 24(9), 2466-2468.

26. Rogers, B. G., et al. (2020). Development of telemedicine infrastructure at an LGBTQ+ clinic to support HIV prevention and care in response to COVID-19, Providence, RI. AIDS and Behavior, 24(10), 2743-2747.

27. Prevention, C.F.D.C.A. (2020). Adjusting the way healthcare services are delivered during the COVID-19 pandemic. Retrieved from https://www.cdc.gov/coronavirus/2019-ncov/hcp/guidancehcf.html\#outpatient-ambulatory.

Publisher's Note Springer Nature remains neutral with regard to jurisdictional claims in published maps and institutional affiliations. 